\title{
Minute gastric carcinoid tumor with regional lymph node metastasis
}

\author{
Toshihiko Shinohara ${ }^{1}$, Shigekazu Ohyama ${ }^{1}$, Hideki Nagano ${ }^{1}$, Nozomi Amaoka ${ }^{1}$, Keitchiro Ohta ${ }^{1}$, \\ Toshiki Matsubara ${ }^{1}$, Toshiharu Yamaguchi ${ }^{1}$, Akio Yanagisawa $^{2}$, Yo Kato ${ }^{2}$, and Tetsuichiro Muto $^{1}$ \\ ${ }^{1}$ Department of Gastrointestinal Surgery, Cancer Institute Hospital, 1-37-1 Kamiikebukuro, Toshima-ku, Tokyo 170-8455, Japan \\ ${ }^{2}$ Department of Pathology, Cancer Institute Hospital, Tokyo, Japan
}

\begin{abstract}
We report a patient with a minute gastric carcinoid tumor with lymph node metastasis, and a small gastric cancer. A 50year-old man having a diagnosis of an elevated lesion on the anterior wall of the gastric body, detected by a series of upper gastrointestinal examinations, was referred to the Cancer Institute Hospital. Careful upper fluoroscopy disclosed a small superficial depressed lesion with converging folds and a superficial elevated lesion covered with nonspecific gastric mucosa. With a final preoperative diagnosis of depressed early cancer and minute carcinoid tumor of the stomach, made by upper gastrointestinal examinations including biopsy, the patient underwent segmental gastrectomy and perigastric lymph node dissection. Histological examination of the resected specimen revealed a lymph node metastasis from a gastric carcinoid tumor of 5-mm diameter, in addition to an early gastric cancer of poorly differentiated adenocarcinoma. Small gastric carcinoid tumors have been regarded as being benign neoplasms biologically. However, the case we present suggests that attention should be paid to the possibility of metastasis at the time of treatment for a minute sporadic gastric carcinoid tumor. We therefore discuss the malignant potential of these tumors, mainly from the viewpoint of histopathological classification, to gain understanding so that the patients can be treated adequately.
\end{abstract}

Key words Carcinoid tumor $\cdot$ Stomach $\cdot$ Regional lymph node metastasis

\section{Introduction}

Gastric carcinoid tumors had been considered to be rare, accounting for about $0.3 \%$ of all gastric tumors and fewer than $2 \%$ of all carcinoid tumors [1,2]. Recently, however, the number of reports of gastric carcinoid tumors has increased, with the widespread use of

Offprint requests to: S. Ohyama

Received: December 11, 2002 / Accepted: August 5, 2003 gastroscopy and improvements in immunohistological techniques.

Rindi et al. [3-5] have characterized three clinicopathologic subtypes of gastric carcinoid tumors. Further, the behavior of any gastric carcinoid tumor depends on its size and depth [6,7].

So far, a minute gastric carcinoid tumor has been regarded as a benign tumor, for which endoscopic excision is recommended. In this report, we describe a case of minute sporadic gastric carcinoid tumor associated with regional lymph node metastasis. We also present a review of the literature.

\section{Case report}

A 50-year-old asymptomatic man was referred to our hospital for further evaluation of an elevated lesion on the anterior wall of the gastric body, detected by a series of upper gastrointestinal examinations. Routine hematologic study revealed no abnormality, and liver and kidney functions were normal. Levels of tumor markers, serum gastrin, and urinary 5-hydroxyindole acetic acid (5-HIAA) were all within normal ranges. Careful gastrointestinal examinations disclosed two lesions, a small elevated lesion with a central depression on the anterior wall of the gastric body, and a superficial depressed lesion in the greater curvature, near the elevated lesion (Fig. 1A,B). Computed tomography (CT) and ultrasonography (US) scans of the upper abdomen showed no metastasis. Biopsy specimens revealed that the depressed lesion was consistent with a poorly differentiated adenocarcinoma and the elevated lesion was a typical carcinoid tumor.

Because of the low probability of lymph node metastasis, and to preserve the stomach function, segmental gastrectomy, together with perigastric lymph node dissection, was performed (The resected stomach is shown in Fig. 2A). Histologically, the depressed lesion 


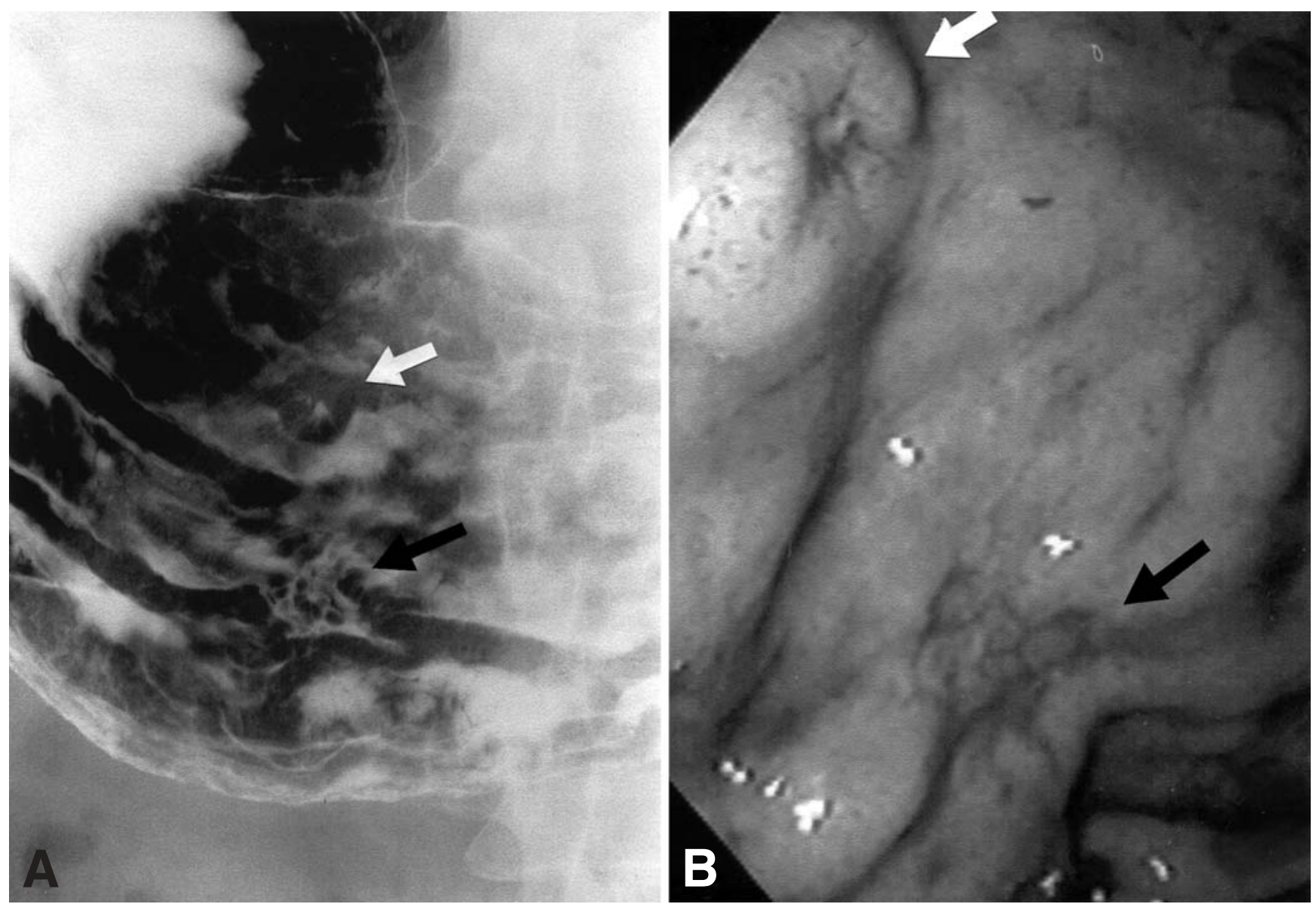

Fig. 1A, B. Gastric fluoroscopy (A) and endoscopy (B) revealed a small elevated lesion with a central depression (white arrow) and a depressed lesion (black arrow) on the anterior wall of the gastric body

consisted of a poorly differentiated adenocarcinoma confined to the mucosal layer (Fig. 2B), and the small elevated lesion, which was $5 \mathrm{~mm}$ in diameter, corresponded to a carcinoid tumor, confined to the submucosal layer, and was characterized by small, microlobular-trabecular aggregates formed by regularly distributed regular monomorphic nuclei (Fig. 2C,D). Immunohistological staining of the carcinoid tumor revealed that it was positive for Grimelius silver and chromogranin. The regional lymph node along the lesser curvature, number 3 according to the $\mathrm{N}$ classification of the Japanese Research Society for Gastric Cancer [8], showed metastasis from the carcinoid tumor (Fig. 2E). No recurrence or metastasis of the carcinoid tumor or adenocarcinoma has been detected during 6 years of follow-up.

\section{Discussion}

Gastric carcinoid tumors had been regarded as rare and benign $[1,2]$. However, with the widespread use of en- doscopy and improvements in immunohistological techniques, recent studies have demonstrated that carcinoid tumors may account for large a proportion as $27.3 \%-$ $41 \%$ of all gastrointestinal endocrine tumors [9-11].

According to Rindi et al. [3-5], gastric carcinoid tumors are classified into three types, on the basis of their clinicopathological features, the presence or absence of hypergastrinemia, and histological features suggesting a benign or low-grade malignant tumor, as follows. Type I includes carcinoid tumors associated with chronic atrophic corporal gastritis; type II includes carcinoid tumors associated with Zollinger-Ellison syndrome or multiple endocrine neoplasia type 1; and type III includes carcinoid tumors associated with sporadic and malignant characteristics without hypergastrinemia. Most gastric carcinoid tumors are mainly composed of enterochromaffin-like cells, and the behavior of any gastric carcinoid tumor depends on its size and depth [6,7].

In Western countries, types I, II, and III were reported to account for $79.6 \%-87.8 \% ; 0-6.3 \%$, and $11.3 \%-14.3 \%$ of gastric carcinoid tumors [3,5,11], 

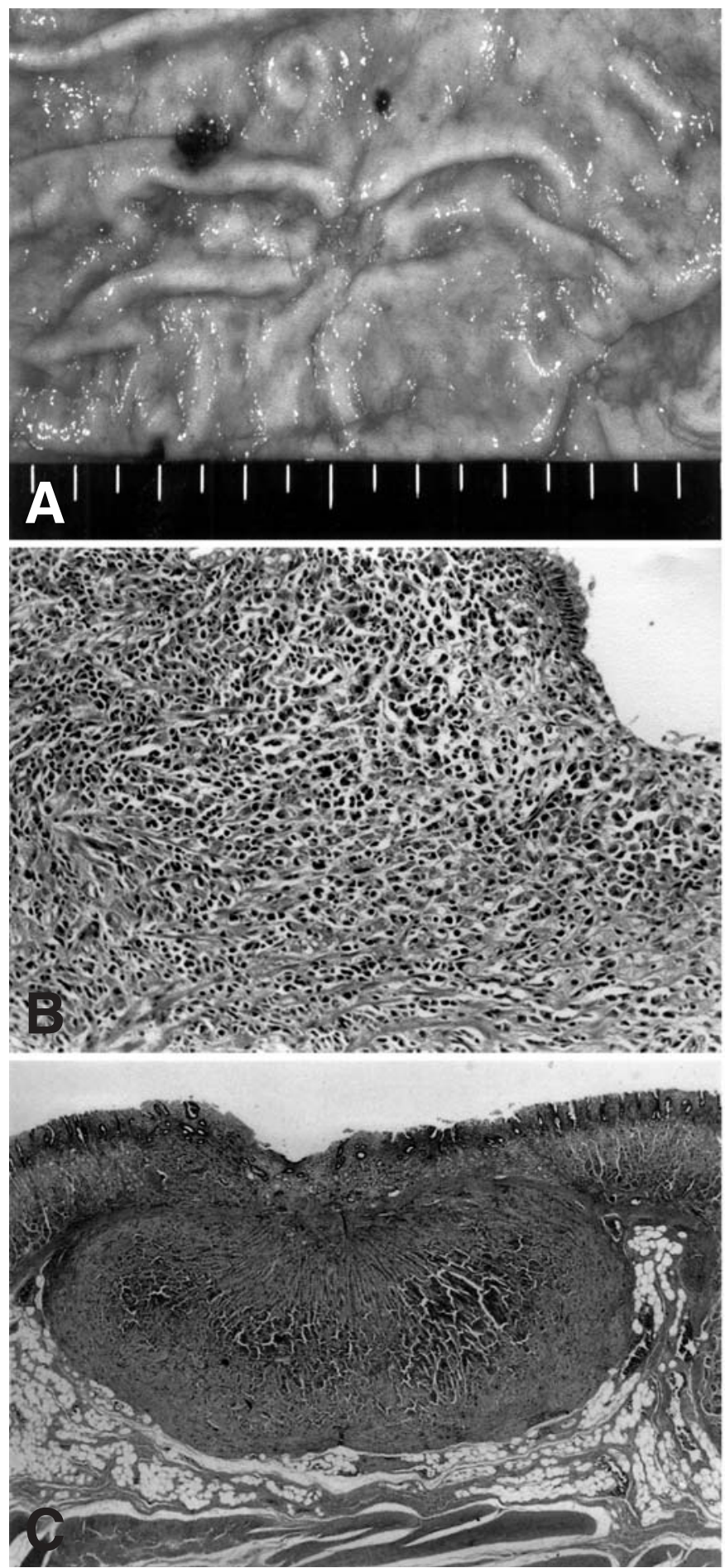

respectively. The rates of regional lymph node metastases from types I, II, and III were reported to be $0-7.6 \%, 0-12 \%$, and $16.7 \%-38.6 \%$ [3,11], respectively.

According to the histology and biological behavior of carcinoid tumors, the decision tree of Gilligan et al. [12] recommends treatments for carcinoid tumors as follows: endoscopic excision followed by endoscopic

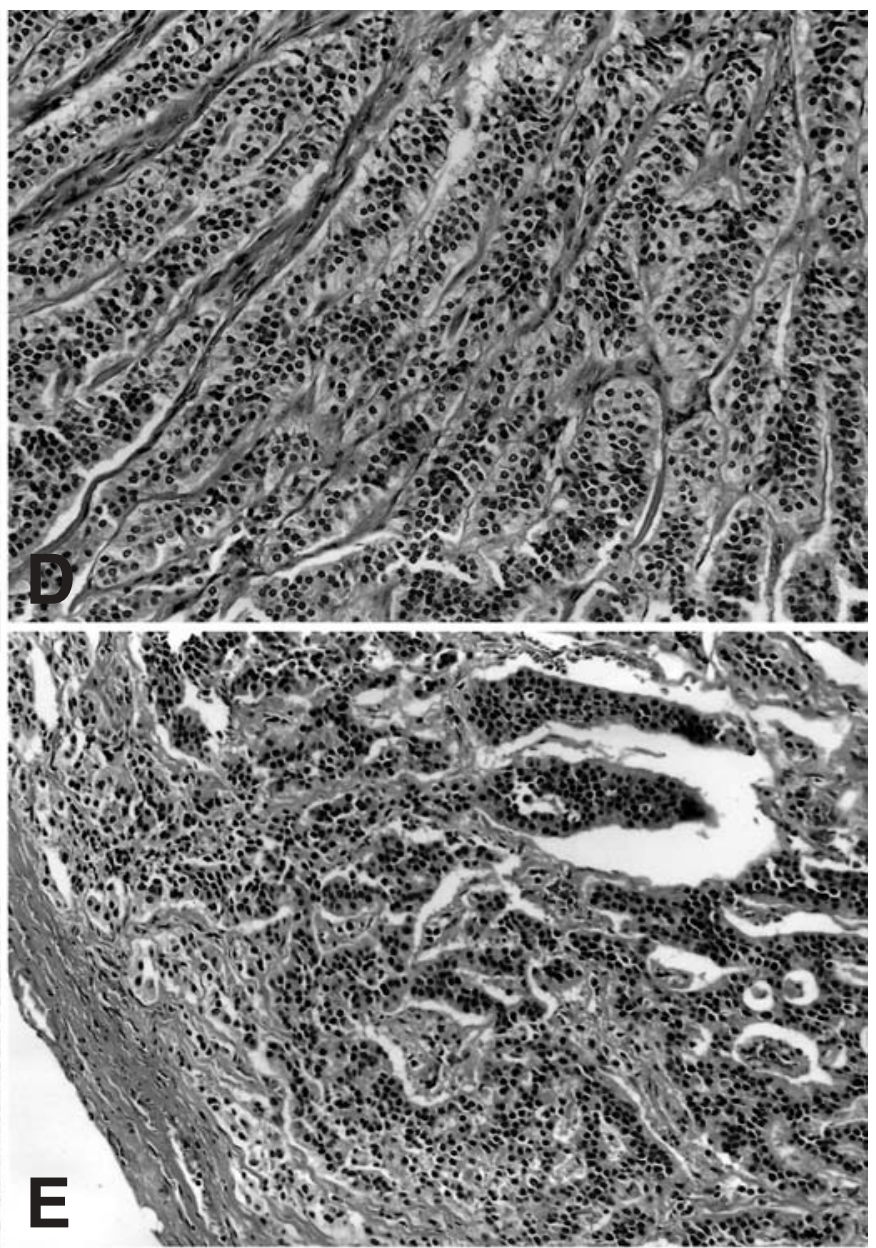

Fig. 2. A Macroscopic appearance of the resected stomach, showing a small elevated lesion with central depression, and nearby, a superficial depressed lesion. B-D Histologically, the depressed lesion consisted of a poorly differentiated adenocarcinoma confined to the mucosa $(\mathbf{B})$, while the elevated tumor, which was $5 \mathrm{~mm}$ in diameter, corresponded to a typical carcinoid tumor, and was confined to the submucosa $(\mathbf{C}, \mathbf{D})$. E The lymph node showed the same histology as the carcinoid tumor, confirming that it was a metastasis of the gastric carcinoid tumor. B H\&E, $\times 50$; C H\&E, $\times 20$; D H\&E, $\times 100$; E H\&E, $\times 100$

surveillance for tumors of types I and II, with the requirement for aggressive surgery for type III.

On the other hand, in Japan, types I, II, and III were reported to account for $34.2 \%-58.2 \%, 0-8.3 \%$, and $33.3 \%-50 \%$ of gastric carcinoid tumors [13,14], respectively. The rates of regional lymph node metastasis from types I, II, and III were $25 \%, 12 \%$, and $35.7 \%$ [13], 
respectively. There are some discrepancies in the histology and biological behavior of gastric carcinoid tumors between the West and Japan.

According to Soga [15], gastric carcinoid tumors are classified into five types, from A to E, according to their histological growth patterns. Types A and B and some of type $\mathrm{E}$ are regarded as ordinary or typical carcinoid tumors, and types $\mathrm{C}$ and $\mathrm{D}$ and some of type $\mathrm{E}$ as atypical carcinoid tumors. The overall metastatic rate was reported to be $33.2 \%$, and there was a significant difference between the typical and atypical groups (29.9\% vs $73.5 \%)$.

In our patient, the carcinoid tumor consisted of microlobular-trabecular aggregates formed by regularly distributed regular monomorphic nuclei (type B according to Soga [15]). Regardless of whether it was a typical carcinoid, however, it was a sporadic tumor with regional lymph node metastasis in the absence of hypergastrinemia, corresponding to Rindi's type III.

The metastatic rates of gastric carcinoid tumors confined within the mucosal, submucosal, and muscular layers were $7.5 \%, 13.2 \%$, and $44.8 \%$, and the metastatic rates by tumor size $(\leqq 10 \mathrm{~mm}, 11-20 \mathrm{~mm}$, and $>20 \mathrm{~mm}$ in diameter) were $8.2 \%, 15.1 \%$, and $52.6 \%$, respectively. Therefore, for gastric carcinoid tumors over $11 \mathrm{~mm}$ in diameter, or those showing massive involvement of the submucosa, en-bloc surgical resection with lymph node dissection is required, while tumors less than $10 \mathrm{~mm}$ in diameter could be managed by endoscopic excision, followed by endoscopic surveillance at 6-month intervals.

In mucosal gastric cancer, limited lymph node dissection with local gastrectomy is appropriate to preserve the stomach function and a reasonably good quality of life [16]. In our patient, a small sporadic carcinoid ( $\leqq 10 \mathrm{~mm}$ in diameter) and early carcinoma of the stomach were evident.

There was no preoperative evidence of lymph node metastasis. Thus, segmental gastrectomy with perigastric lymph node dissection was performed. Synchronous malignancy was found in $12 \%$ of the patients with gastric carcinoid tumors reported in the literature, and nearly half of them were found to have early gastric carcinoma. Of these patients with synchronous malignancy, almost half exhibited the coexistence of two histological elements, carcinoid tumor and adenocarcinoma, in single neoplastic nodules, as either composite or collision tumors [15].

Previous studies showed that type III tumors were mostly solitary, larger than $11 \mathrm{~mm}$ in $25 \%-70 \%$ of patients, and deeply invasive (infiltrating into the muscular layer and serosa in 77\% of patients). Accordingly, these tumors have a high metastatic tendency $(36 \%-$ $39 \%)[11,17,18]$.

Recently, we have found some reports in the literature describing type III tumors that were less than
$10 \mathrm{~mm}$ in diameter with regional lymph node metastasis $[19,20]$. Clinicopathological analysis showed that such type III tumors had a higher Ki67 index (suggesting high proliferation of tumor cells) and greater p53 accumulation (suggesting mutational transformation) than type I and II tumors [21,22]. From these findings, type III gastric carcinoids can be regarded as having considerable malignant potential, regardless of their size $[23,24]$.

We have described a case of small, sporadic gastric carcinoid tumor with regional lymph node metastasis, corresponding to Rindi's type III. We emphasize that type III gastric carcinoid tumors require aggressive surgical treatment with lymph node dissection, even if they are smaller than $10 \mathrm{~mm}$ in diameter.

\section{References}

1. Godwin JD. Carcinoid tumors: an analysis of 2837 cases. Cancer 1975;36:560-9.

2. McDonald RA. A study of 356 carcinoids of the gastrointestinal tract. Am J Med 1956;21:867-78.

3. Rindi G, Luinetti O, Cornaggis M, Capella C, Solcia E. Three subtypes of gastric argyrophil carcinoid and gastric neuroendocrine carcinoma: a clinicopathologic study. Gastroenterology 1993;104:994-1006.

4. Rindi G. Clinicopathologic aspects of gastric neuroendocrine tumors. Am J Surg Pathol 1995;19(Suppl 1):s20-9.

5. Rindi G, Bordi C, Rappel S, La Rosa S, Stolte M, Solcia E. Gastric carcinoids and neuroendocrine carcinomas: pathogenesis, pathology and behavior. World J Surg 1996;20:168-72.

6. Moertel CG. Treatment of carcinoid tumor and the malignant carcinoid syndrome. J Clin Oncol 1983;1:727-40.

7. Hoberock TR, Knutson C, Polk HC. Clinical aspects of invasive carcinoid tumors. South Med J 1975;68:33-7.

8. Japanese Research Society for Gastric Cancer. The general rule for gastric cancer study in surgery and pathology. Jpn J Surg 1981;11:127-45.

9. Modlin IM, Goldenring JR, Lawton GP, Hunt R. Aspects of the theoretical basis and clinical relevance of low acid states. Am J Gastroenterol 1994;89:308-18.

10. Mizuma K, Shibuya H, Totsuka M, Hayasaka H. Carcinoid of the stomach: a case report and review of 100 cases reported in Japan. Ann Chir Gynaecol 1983;72:23-7.

11. Rappel S, Altendorf-Hofmann A, Stolte M. Prognosis of gastric carcinoid tumors. Digestion 1995;56:455-62.

12. Gilligan CJ, Phil M, Lawton GP, Tang LH, West AB, Modlin IM. Gastric carcinoid tumors: the biology and therapy of an enigmatic and controversial lesion. Am J Gastroenterol 1995;90:338-52.

13. Iwashita A, Takayama S, Oishi Y, Iwai K, Yao T, Shimoda T, et al. A clinicopathological study on gastric carcinoid tumors: with special reference to difference of nodal metastasis between tumors with type A gastritis and those with non type A gastritis (in Japanese with English abstract). I to Cho (Stomach and Intestine) 2000;35:1365-80.

14. Iwafuchi M, Watanabe H, Noda Y, Ajioka Y, Enjozi M, Ito M. Gastrointestinal carcinoid tumors in the Japanese: incidence and characteristics based on anatomic classification, with special reference to difference between carcinoid tumor and endocrine cell carcinoma (in Japanese with English abstract). I to Cho (Stomach and Intestine) 1989;24:869-82.

15. Soga J. Carcinoid tumors: a statistical analysis of a Japanese series of 3126 reported and 1180 autopsy cases. Acta Med Biol 1994;42: 87-102. 
16. Tsujitani S, Oka S, Saito H, Kondo A, Ikeguchi M, Maeta M, et al. Less invasive surgery for early gastriccancer based on the low probability of lymph node metastasis. Surgery 1999;125:14854.

17. Bordi C, Yu JY, Baggi MT, Davoli C, Pilato FP, Baruzzi G, et al. Gastric carcinoids and their precursor lesions. A histologic and immunohistochemical study of 23 cases. Cancer 1991;67:66372.

18. Stolte M, Ebert D, Seifert E, Schulte F, Rode J. Zur Prognose der Karzinoidtumoren des Magen darm. 1988;5:246-56.

19. Kumashiro R, Naitou H, Teshima K, Sakai T, Inutsuka S. Minute gastric carcinoid tumor with regional lymph node metastasis. Int Surg 1989;74:198-200.

20. Morise K, Kusugami K, Hayakawa M, Nakata S, Inagaki T, Hayashi N, et al. Minute gastric tumor of the stomach: report of two cases and review of the Japanese literature. Gastroenterol Jpn 1985;20:596-603.

21. Rindi G, Azzoni C, Rosa SL, Klersy C, Paolotti D, Rappel S, et al. ECL cell tumor and poorly differentiated endocrine carcinoma of the stomach: prognostic evaluation by pathological analysis. Gastroenterology 1999;116:532-42.

22. La Rosa S, Sessa F, Capella C, Riva C, Leone BE, Klersy C, et al. Prognostic criteria in nonfunctioning pancreatic endocrine tumors. Virchows Arch 1996;429:323-33.

23. Ahlman H, Wangberg B, Nilsson O, Grimelius L, Granerus G, Modlin M, et al. Aspects of diagnosis and treatment of the foregut carcinoid syndrome. Scand J Gastroenterol 1992;27:459-71.

24. Capella C, Heitz PU, Hofler H, Solcia E, Kloppel G. Classification of neuroendocrine tumors of the lung, pancreas and gut. Digestion 1994;55 (Suppl 3):11-23. 